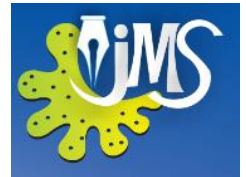

\title{
'Translating the Untranslatable': An Analysis on Translating Culture Specific References in Translating a Script for Dubbing Purpose
}

\author{
J.A.M. Buddhima Karunarathna \\ Department of Language Studies, University of Vocational Technology, Sri Lanka
}

\begin{abstract}
The field of translation is becoming exceedingly popular with the advancement of technology. In the Sri Lankan context, the field of audio visual translations, specifically dubbing and subtitling was first practiced in early 1980s. However, compared to the practices in the field of audio visual translation in the other parts of the world, the advancement of the field in Sri Lanka was comparatively slow. Nevertheless, dubbed dramas, movies, etc. from various continents of the world are dubbed in Sinhala and Tamil and telecast in Sri Lanka. Translating a script for dubbing purpose is challenging since the translator has to focus on the linguistic factors as well as socio-cultural factors related to both the source language and the target language. The purpose of the study was to analyze strategies in translating culture specific expressions in translating a script for dubbing purpose. As the methodology, culture specific references of a Korean drama which was translated and telecast in Sinhala was analyzed. The analysis was done from the word level and above word level considering the linguistic factors as well as socio-cultural factors of both source language and the target language. Thus, the findings show that apart from strategies of translating the other conventional media, polysemiotic nature in audio visual translations, has led the translator to use several interventional strategies in translating culture specific expressions within the cultural, linguistic, technical and contextual considerations in translating a script for dubbing purpose, challenging the conventional theories of translation equivalence.
\end{abstract}

KEYWORDS: Audio visual translations, dubbing, culture specific references, source language, target language

Corresponding author: J.A.M. Buddhima Karunarathna, Email: write2buddhima@yahoo.com 


\section{INTRODUCTION}

Translation is the process of translating words or texts from one language to another. The word 'translation' has several meanings; the general subject field or phenomenon, the product and the process of producing the translation. The process of translation between two different written languages involves the changing of an original written text, which is identified as the source text (ST) in the original verbal language; the source language (SL) into a written text; the target text (TT) in a different verbal language i.e. the target language (TL).

Audiovisual translation (AVT) is translating of any material in audio, visual or audiovisual format. Dubbing, subtitling, localization and media accessibility (audio description, subtitling for the deaf and hard of hearing) are some of the most common techniques of AVT. Dubbing is the process of translation, adaptation and recording of a video to create the feeling of illusion that the characters 'talk' in the target language. Dubbing raises a number of theoretical and practical issues, such as cultural appropriation, narrative manipulation, censorship, lip and temporal synchronization, reception and tolerance of dubbing, synchronization between verbal and non verbal elements, etc. Dubbing has on the whole been relatively little studied, probably to some extent because of the division of labor between the translator, the adapter and the dubbing artist, and the responsibilities that it implies, and partly also because any analysis entails a considerable initial effort of transcription.

In the Sri Lankan context, AVT, specifically dubbing and subtitling was first practiced in early 1980s. Currently, dubbed dramas, drama series, movies, documentaries, etc. from various continents of the world are dubbed in Sinhala or Tamil and telecast by almost all the television channels. These programmes, especially Korean dramas have become very popular among the Sri Lankan audience at present. But due to the complexity of the field of dubbing and various factors like the high amount of cost for a dubbing production, lack of human and technical resources available in the country, the field of dubbing has not developed in Sri Lanka, compared to other countries who telecast AVT products based on dubbing.

Thus, in the Sri Lankan context, the field of audio visual translations has not flourished in par with the international context yet. Hence, a limited number of researches are being conducted regarding the subject. Increasing numbers of dubbed drama programmes being telecast in Sri Lanka, and matters arising from them, intensify the need of having empirical studies to solve the practical problems which occur linguistically, culturally, socially, technically, etc. This research is an attempt to fill the research gap that exists in the field of audio visual translations in the Sri Lankan context, with a special focus on culture specific references (CSRs) of SL and TL. This research is based on the hypothesis; without paying attention to culture of the TL, there won't be culturally acceptable AV translated product among the target audience. Thus, the objectives of the research would be to study the strategies that are used in translated dubbing product in the international context and to propose strategies that can be used in translating a script for dubbing purpose in the Sri Lankan context.

\section{LITERATURE SURVEY}

AVT is a specialized branch of translation studies which implies the presence of any kind of audio and visual electronic system in the translation process. There is a vast range of audiovisual products available to translate such as television programmes, feature films, theatrical plays, musicals, web pages, video games, etc. Though verbal and visual codes in audiovisuals are linked to an extent that the words naturally tend to rely on the visuals, the translation in AV products operate on a verbal level alone. 
Since AV materials are meant to be seen and heard simultaneously, their translation is different from translating print. Written works are primarily meant to be read. Illustrations in books, newspapers, journals, and magazines such as photographs, diagrams, and graphs are there to support and improve the verbal content. On the other hand the verbal and visual contents of $\mathrm{AV}$ products function inseparably to create a meaningful whole.

To generate a single effect, audiovisuals are made up of numerous codes that interact with each other. There are different levels of interactions as well. On one level, audiovisual products contain a series of verbal messages that will be perceived both acoustically and visually. In filmic products, as well as what the actors say, audiences may also hear the lyrics of songs while simultaneously being exposed to a range of written information such as street signs, billboards, letters, notes, etc. Also, at the beginning and end of a programme, substantial written information about it, such as the names of the director, producers, the cast, and the production team will also be visible.

On a different level, but together with such acoustic and visual verbal input, filmic products also contain nonverbal sound effects and background noises, body sounds (breathing, laughter, crying, etc.) and music. Equally, and an additional meaning is conveyed through the facial expressions of the actor, gestures and movements, costumes, hairstyles, makeup, etc. Furthermore, scenery, colors, special effects, and three dimensionality are also part of the filmic whole. Thus, screen products are considered polysemiotic; which are made up of numerous codes that interact to produce a single effect.

Thus, Chiaro (2013) argues "AVT needs to take all this diversified verbal and visual information into account bearing in mind that this inseparable link between verbal and visual codes may often constrain the translation process."

Delabastita (1989) highlights the specific characteristics of AVT characteristics, and stresses that film is "a multi-channel and multicode type of communication." He focuses on the two channels in film communication; the visual and the acoustic. According to Delabastita (1990), film is a complex meaningful sign consisting of a multitude of codes. The codes are the verbal (including various dialects, sociolects); literary and theatrical (storyline, dialog); proxemic and kinetic (i.e., referring to non-verbal performance); and cinematic (techniques, genres). Further, Delabastita points out the four basic elements that define the audiovisual text and establish a basis for its semiotic texture: as a) Verbal acoustic ( dialogue, monologue, songs, voice-off), b) Nonverbal acoustic: musical score, sound effects, noises, c) Visual-nonverbal: image, photography, gestures, d) Visual-verbal: inserts, banners, letters, messages on computer screens, newspaper headlines. (Diaz Cintas, 2008)

Thus, polysemiotic nature of $\mathrm{AV}$ product is challenging for a translator in the process of translation. Chiaro (2005) advocates that there are four basic steps involved in the process of dubbing a programme from start to finish; translating the script, adaptation to sound, recording the script through dubbing artist, and mixing into the original recording. By tradition, the dubbing translator need not be proficient in the SL, but knowing it would be an advantage. However the dubbing translator should be creative and talented enough in the TL to create fresh dialogue that is convincing. As well as rendering talk natural, care is taken to ensure that the dialogue fits into visual features on screen such as lip movement, facial expressions, etc. Furthermore, the new dialogue also needs to take the emotive content of each utterance into account. Chaume (2006) puts forward the fact that, with the awareness that a thorough understanding of the ST is a crucial asset for a translator, it is becoming ever more common for the two processes of the translation itself and the adaptation to merge and be carried out by a single translator who is proficient in both languages. However, the challenge is to write a dubbing script and record the dialogues in such 
way that the dubbing sound track is lipsynchronous with the visual acting on screen and the content of the original dialogues is transferred to TL.

Chiaro (2005) finds that both dubbing translators and subtitling translators have to contend with three basic categories of translational hurdles: 1.highly culture-specific references (e.g. place names, references to sports and festivities, famous people, monetary systems, institutions, etc.); 2. language-specific features (terms of address, taboo language, etc.); 3. areas of overlap between language and culture (songs, rhymes, jokes, etc.).

Gambier (2007) suggests that dubbing has on the whole been relatively little studied, because of the division of labor between the translator and/or the adaptor, the actors, the responsibilities that it implies, and also because any analysis entails a considerable initial effort of transcription. Nevertheless, dubbing raises a number of theoretical and practical issues, such as cultural appropriation, narrative manipulation, censorship, lip synchronization, reception and tolerance of dubbing, synchronization between verbal and non-verbal elements (gestures, facial expressions, gaze, body movements, etc.).

Further, Gambier (2007) points out "very few systematic studies have examined the production and reception or the cultural and linguistic impact of audio visual translation." $\mathrm{He}$ points out that two factors explain this paradox; the linguistic and literary background of most researchers and the constraints of printed publication in two dimensions.

However, viewers' acceptance is often stretched to extreme limits, especially because of the presence of a series of highly culture-specific references (CSRs). CSRs are entities that are typical of one particular culture and that culture alone, and they can be either exclusively or predominantly visual, exclusively verbal or else both visual and verbal in nature.
Studying US filmic products, Antonini and Chiaro (2005) have identified ten areas in which what they have labelled 'lingua-cultural drops in translational voltage' may occur in: 1 . institutions, 2. educational references, 3. place names, 4. units of measurement, 5. monetary systems, 6. national sports and pastimes, 7. food and drink, 8. holidays and festivities, 9. books, films and TV programmes, 10. celebrities and personalities. 'Lingua-cultural drops in translational voltage' refer to the inevitable perceived uneasiness and turbulence in the verbal code with respect to the visuals among the target audience.

\section{MATERIALS AND METHODS}

This research was based on qualitative method. The research was conducted using both primary data and secondary data. As for the primary data, the scripts of the Korean drama "Maai" which was translated into Sinhala and telecast as "Isiwara Wedaduru" were analyzed discourse wise i.e. at the morphological level and above morphological level, in relation to CSRs. The selected drama was telecast by Sri Lanka Rupavahini Cooporation in 2013/2014. As for secondary data, library research was conducted to find out the strategies used in translating CSRs in AVT.

Korean drama series "Maai" was telecast by MBC (Munhawa Broadcasting Cooperation) in South Korea during 2012/2013. The drama consists of 50 episodes of dramas with 60 minutes for each episode. The drama was directed by Lee Byung-Hoon and script was written by Kim Yi-Young. The genre of the series is historical drama and medical drama.

The genre of "Isiwara wedaduru" is family drama. The dubbed programme was directed under the supervision of Athula Ransirilal and directed by Amali Wijesekara and Thusitha Vidanapathirana. The script was translated by the researcher herself. Apart from the script of the SL, the script used for subtitling in English 
which was used in translating as an aid was also used in the research as a tool.

It can be argued that, by selecting a sample translated by the researcher would lead to personal biasness of the study. But, it is due to multidisciplinary nature of research in audio visual translations. Because, in carrying out a study regarding linguistic perspective in dubbing, a researcher should be familiar with the SL and the TL.

Usually in the Sri Lankan context, a script is translated for dubbing in two ways. One is a translator translates the script that is being given in SL or English language (if the translator is not familiar with the SL and/or if a script is available in English) and then there's an adaptor to convert it into a lip synchronized script. The second way is; translation and lip synchronizing is done by the same person, i.e. lip synchronized script translator.

Considering the notion of translation equivalence and shift, the second way of practice of translation mentioned above, makes less interference to the SL when it is translated to the TL. Since it will support in keeping the originality of the SL, and the researcher is familiar with both the SL and the TL, the researcher could do a more in-depth analysis regarding translating CSRs of the selected sample.

\section{RESULTS AND DISCUSSION}

The historical background of the source programme is set on the life of a Joseon-era lowclass veterinarian specializing in the treatment of horses, who rises to become the royal physician in charge of the King's health. Joseon was a Korean kingdom founded by Taejo Yi Seonggye that existed from July 1392 to October 1897. Thus, historical and cultural background of the source programme was subjected to an in depth study to make it authentic for the Sri Lankan audience.

\subsection{Medicine and Related References}

The title of the source programme is "Maai", whereas in English MBC refers as "Horse Doctor" and/or "King's Doctor". "Maai" is a term used during the Joseon Period to describe a veterinarian, who was regarded as socially inferior. In Sinhala "Isiwara wedaduru", was suggested by the dubbing director considering the factors of the TL and the culture, not referring to the veterinarian title given in the SL. Because in the Sri Lankan context, the role of the veterinarian is respected as well as a physician throughout the history up to now, unlike in the source culture.

During the Jeson period of veterinarians ("maai") and other commoners belonged to lower class ("chomin") and the physicians ("isha") and other aristocratic belong to high class ("yanban"). But in the target culture, it was not the class system which is prominent but the caste system. Thus, for the word physician ("isen") the term "wedaduru" was selected and for the word veterinarian ("maai") the term "wedana" was selected. In the TL both terms refer to a physician. To keep the difference of the two terms throughout the dubbed programme, in the first lip synchronized translated episode, the terms "as wedaa", "ashwa wedana" were used in introducing veterinarians.

Since the story is based on a veterinarian who rises to the royal physician and the first surgeon of the medical history of Korea, there were specific references related to oriental Jeson medicine. Therefore, in translating such references, words used in standard modern TL couldn't be used considering the setting of the source programme and the SL which is based on historical discourse.

Lip synchronized translations for such medically related references in the TL can be discussed under medicinal tools, acupuncture points, medical books, body organs, ailments, symptoms, and medical prescriptions. 
Some such references were directly translated into the TL and some were directly borrowed from SL to TL. The names of tools and the ailments could be translated by using the available medical jargon of the oriental medicine of the TL but references for acupuncture points and prescriptions were directly borrowed from the SL. References related to acupuncture points and prescriptions were directly borrowed because such practices and references do not exist in the culture of the target audience.

Moreover, especially in reference to the medical books, names of some books were adapted and names of some books in the source programme were omitted. The reason for adaptation is that these books are unique to the oriental medicine of the source culture. In the attempt of adapting the names, the content of the book was noted and the adaptations were done accordingly. However, in the attempt of adaptation, the length of each utterances surpassed, so that some of the names of the books were totally omitted. Because of the polysemiotic nature of an audio visual product, some of the names of the books could be read by the audience if the audience is familiar with the SL when the books are shown.

\subsection{Relationships}

In translating expressions related to relationships, the culture of both SL and the TL were considered.

In the SL, different people use different titles to address each other, and in the case of direct translation to the TL, it would create a confusion in the target audience. For example; in the work place, people are called by their surname and job title and at home everyone has their own title which can change depending on who they are talking to. Relationship to the speaker, rank, age, or gender can all play a part. Though only some of these terms are used in contemporary society of the source culture.

Especially in translating the relationships related to courtroom forms of address, legal topography, agents related to different institutions, etc. different strategies had to be used.

In translating the kinships in the royal court, the king, the queen, queen mother, Princess Sukhi were addressed by each other and how they address the others in different situations were translated focusing on the cultural reasons for different utterances and the restrictions of the length of the utterance. The term "yong-gam" used to address high ranking noblemen was translated as "amaathyathuma", "mathithuma" and "pradhnithuma" depending on colour of the uniform they wear. The term "nauri" which is used to address middle ranking men was translated as "nildarithuma". The eunuchs of the palace were continued to be addressed as "paalakathuma" whereas the target audience would find it offensive if it is translated according to the meaning and the practice of the SL.

To note the difference between yanbans and chomins, the way of addressing family relationships contributed a lot. Thus to call elder brother, the terms were translated in to TL as "aiya" and "aiyandi" respectively for lower class characters and higher class characters. Similarly, in addressing the father, the terms "appachchi" and "piyaanani" were used and in addressing uncles, the terms "maama" and "maamandi" were used to make the TL audience aware of the class difference prevailed in the culture of the SL.

Further, to add more meaning in the TL programme when referring to toddlers and children by adults, words like "kirikatiya", "daru patiya", "daruwa" were used, even though such terms from the SL were not used.

Moreover, in fights and arguments, the offensive and taboo words to refer to each other were omitted in the SL. Instead direct translations for such, words like "ohe" from the TL were applied. 


\subsection{Food}

Though the selected sample programme is based on medicine and its practices, there are occasions that it refers to food.

However, following are 02 examples from the translated script, which the translator has practiced when a mismatch arises between the food cultures of the SL and TL.

English meaning of a dialogue from the source programme of Episode 2, Scene 5 is as follows;

Kwanghyun: Who catches fish in the Capital? They eat grilled beef!

Children: Grilled beef? What's that? Kwanghyun: Idiots! Don't ask such questions in the Capital. They'll know you're rustics. Grilled beef; first they slice meat of a cow.

Children: Really!

Kwanghyun: Then they marinate in it spices. Children: Really!

Kwanghyun: Then grill it in an open flame. It's very tasty!

The above dialogue is translated to TL as follows;

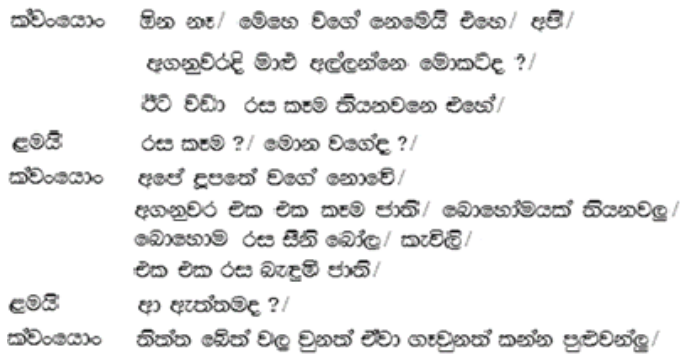

The meaning of the translation is totally different from the original dialogue. The reason is that in the Sri Lankan context, though the habit of eating beef is there, it is not considered in a glorifying manner. Thus, the utterances should be rephrased according to the gestures acted out by the character Kwanghyun and other children.
Similarly, English meaning of a dialogue from the source programme of Episode 5, Scene 30 is as follows;

Servant: Isn't that the sick puppy? Kwanghun, I thought to have soup after this puppy dies.

Kwanghun: Auh, dream on. Now this puppy won't die. I healed him!

Servant: Huh!

The dialogue is translated to TL as follows;

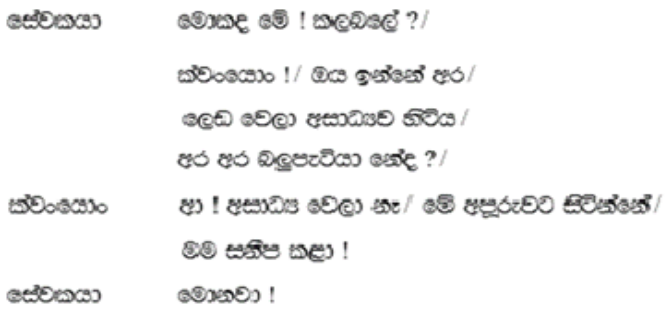

The meaning of the translation here too is totally different from that of the SL. In the culture of the SL, eating flesh of dogs is accepted and practiced. This practice is totally unacceptable in the target audience. Thus, there can be a rejection of the total dubbed programme among the target audience, if it is translated as it is.

It can be argued since it negatively affect the originality of the source programme, rather than recreating the dialogues it would be better to remove that part from the dubbed programme itself. But, when it comes to dubbing, since it has unique features which are interrelated as visual and verbal, such attempts badly affect the sequence of the programme. Thus, recreating the dialogue in the TL was the best possible strategy here.

\subsection{Jokes}

Humour is extremely difficult to translate especially when visuals and vocals amalgamate.

Most of the jokes in the sample programme are uttered by the character of Jabon, supported by the character of Kibae. Most utterances in jokes are considered as taboos in both cultures of the 
SL and the TL. Such jokes are related to references of sexual connotations. Thus, instead of removing such scenes from the dubbed programme, the dialogues are being recreated according to the gestures made by the characters. Sexual connotations were totally omitted in the translation.

This supports the argument of Chiaro (2006), "Given that it is highly unlikely to find the same words, sounds, forms and concepts in two different languages which must also happen to possess the same ambiguity that can be exploited for humorous means, this solution is extremely difficult and very much dependent on the dexterity of the translators and dubbingscriptwriters". Regarding translating comedies in audio visual products Chiaro has specifically mentioned that "In a comedy, which may well rely on several of these features concurrently in order to create the desired effect, if the verbal code is the only dimension which can be manipulated to aid the target culture in capturing the humour, the translator's job is a delicate one" (Chiaro, 2006).

\subsection{Multilingual Scenes}

Multilingual scenes contain characters speaking in a language other than SL. Though the language of the source programme is essentially Korean, because of the international relations took place during the Jeseon dynasty, specifically with China, there are scenes, though very few, which are being spoken in Chinese language as well. Some utterances are uttered by the major characters and some by the supportive characters within the boundaries of Jeseon when the Chinese visit Jeseon or else when they visit China for different purposes. Such utterances were translated by referring to the English subtitled script forwarded by the MBC.

There are dialogues in Chinese language in Episode 5, Episode 30, Episode 31 and Episode 32. Thus, the meanings were translated to suit the lip synchronization, assuming that the target audience would comprehend the non-verbal clues of the Chinese dress code of the contemporary era.

\section{CONCLUSION AND FURTHER WORK}

Translating CSRs from the SL to TL in an AV production basically depends upon the choice of the translator. The choice of the translator is based on the translator's sensitivity towards the culture of the source audience and the culture of the target audience. Strategies used in AVT as omission, literal translation, borrowing, equivalence, adaptation, replacement, rephrasing, replacement, etc. should be practiced in a balanced manner. For this purpose, AVT can be considered in a holistic sense of linguistic translation and cultural translations.

Hence, the strategies can be discussed in three aspects as; orientation to the source culture, orientation to the target culture and then neutralization according to the context. In a dubbed programme, the speech of the source and the target programmes is situated in a range between spontaneous and planned discourse, displaying features of both extremes. Dubbing must attempt to sound spontaneous and natural, as if it were spoken speech, whilst actually being planned. The use of these aspects depend on a particular scene, the preference of the translator, the codability of the linguistic and cultural items, and the availability of local substitutes. However the selection is governed by norms. It varies with the genre, target culture, censorship, political issues, patronage and marketing concerns. Because norms and principles valued by the target audience and practiced by the institution of dubbed production should be considered in AV translations. Different sociological variables of the source audience such as; age, level of education, reading aptitudes, command of foreign languages, hearing/sight difficulties and audio visual variables: i.e. broadcasting time, types of TV channels, film genre, interplay images/dialogues, etc. should also be taken into account in this regard. 
However these aspects will never be total because the dubbed programme as the embodiment of foreign culture is the focus of attention and is present at all times, along with the coexistence and contestation between the two worlds of the SL and the TL. Thus, the notion of equivalence in theories of translation is also challenged in AVT, since the focus is based on the reception of the target audience, which is debatable regarding the originality of the source programme.

AVT is unique in the sense that its complexity and restrictions greatly exceed other types of translations. The audiovisual translator feels constrained by the medium of communication and is subject to textual, multimedial and multimodal restrictions that limit the rendering of the source text into the target text. A comprehensive examination and analysis of the basic concepts in AVT theory will not only assist the researcher and the translator in the process of translation but also provide a basis for better understanding of the developing theories in AVT.

Moreover, though the research is based on the role of the lip synchronized translator in dubbing, the translated script can subjected to changes by the dubbing director, producers and even by the dubbing artists while dubbing. Nevertheless, cultural sensitivity and creativity in the resource persons involved in an AVT product provide opportunities for a successful and acceptable product.

Thus, AVT is unique because of its restrictions and complexity. Even though the discipline of translation studies itself has received a lot of critical attention, theories and basic concepts of audiovisual translation has yet to be widely known or regarded as integral to its analysis. In the Sri Lankan context, there's a vast gap in the field of AVT regarding almost all the aspects of it. The most popular aspects of AVT are dubbing and subtitling, though many researches are not conducted. However, further AVT research could be extended not only to cultural and linguistic factors but also social and economic factors as well. Further, the AVT research areas can be extended to the technical and technological perspectives in audio visual translations as well.

\section{ACKNOWLEDGEMENT}

Mr. Athula Ransirilal, Director, Film and Dubbing Unit, Sri Lanka Rupavahini Cooperation.

\section{REFERENCES}

BAKER M. The Status of Equivalence in Translation Studies: An Appraisal. EnglishChinese Comparative Study and Translation. Zijian (ed.). Shanghai: Foreign Languages Education Press. 2004.

CHIARO D. Audio visual Translation, The Encyclopedia of Applied Linguistics, Chapelle (ed), Oxford: Blackwell Publishing; 2013. doi: 10.1002/9781405198431.wbeal0061

CHIARO D. Issues in Audiovisual Translation. Routledge Companion to Translation Studies. Ed. Jeremy Munday. London: Routledge. 2010.

DIAZ-CINTAS. Introduction: The Landscapes of Audiovisual Translation. The Journal of Specialized Translation, Issue 6. 2006.

DIAZ-CINTAS. In search of a theoretical framework for the study of audiovisual translation. Topics in Audiovisual Translation. Pilar (Ed.). Amsterdam/Philadelphia: John Benjamins. 2004.

GAMBIER Y. Handbook of Translation Studies; Challenges in research on audiovisual translation. Gambier, Y \& Doorselaer, editors; John Benjamins. 2014.

HOLMES JAMES S. The name and nature of translation studies, in Lawrence Venuti (Ed.) 
The Translation Studies Reader, 2nd edition; Routledge. 2004.

KULARATNE JASN. Problems faced by the translators in Audio-Visual Translations; MA thesis, University of Kelaniya. 2009.

PANOU DESPONIA. Theory and Practice in Language Studies: Equivalence in Translation Theories: A Critical Evaluation, Vol. 3, Academy Publisher, Finland. 2013.

SENEVIRATNE C. Difficulties encountered by a translator engaged with a process of dubbing translation. (A linguistic approach giving preference to phonological problems and difficulties in translating culture specific concepts); MPhil thesis, University of Kelaniya. 2013.

\section{TAYLOR \& FRANCIS.} www.routldgtranslationstudiesportal.com, 2013. http://cw.routledge.com/textbooks/translationstu dies/data/samples/9780415584890.pdf Accessed 11 July 2015. 